\title{
Uso de bacteriófagos en gallinas de postura infectadas con Salmonella enterica serotipo Enteritidis: prevención de la colonización intestinal y reproductiva"
}

\author{
Bacteriophage use in laying hens infected with Salmonella enterica serovar \\ Enteritidis: prevention of intestinal and reproductive colonization

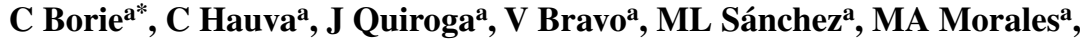 \\ P Retamal $^{a}$, J Retamales ${ }^{b}$, J Robeson ${ }^{b}$ \\ aDepartamento de Medicina Preventiva Animal, Facultad de Ciencias Veterinarias y Pecuarias, \\ Universidad de Chile, Santiago, Chile. \\ bLaboratorio de Bacteriología, Instituto de Biología, Pontificia Universidad Católica de Valparaíso, \\ Valparaíso, Chile.
}

\begin{abstract}
SUMMARY
Salmonella enterica serovar Enteritidis (SE) remains an important enteropathogen in the poultry industry and public health. Due to the limited effectiveness of control measures, lytic bacteriophages have shown a potential use as biocontrollers of SE in birds. The aim of this study was to determine the effectiveness of prophylactic therapy with phages to control intestinal and reproductive tract colonization of SE in laying hens. 22-week old HyLine Brown hens free of Salmonella, were treated with a mixture of three bacteriophages $\left(10^{11} \mathrm{PFU} / \mathrm{dose} / \mathrm{phage}\right)$ and challenged with $2.4 \mathrm{x} 10^{8} \mathrm{CFU}$ of SE, 24 hours post phage treatment. On day 10 post challenge, hens were euthanatized, and individual samples of cecum, ovary and oviduct, were analyzed using qualitative and quantitative bacteriology. Eggs laid during the experience were collected and processed to detect SE. The incidence of Salmonella in ceca was similar between positive control and treated groups $(96.67 \%)$ and cecal bacterial counts did not present significant differences between them $(\mathrm{P}>0.05)$. In reproductive tissues, phagetherapy was able to slightly reduce the $\mathrm{SE}$ count in the ovary $(\mathrm{P} \leq 0.05)$, but not in oviducts $(\mathrm{P}>0.05)$. This lytic activity of phages observed in ovaric tissue, encourages further efforts to elucidate the real contribution of bacteriophages as SE biocontrollers in laying hens.
\end{abstract}

Palabras clave: Salmonella, prevención, bacteriófagos, gallinas de postura, aves. Key words: Salmonella, prevention, bacteriophages, laying hens, poultry.

\section{INTRODUCCIÓN}

Las infecciones por Salmonella spp corresponden a una enfermedad transmitida por alimentos, de fuerte impacto en la salud pública. En la Unión Europea, durante el año 2008, la salmonelosis fue la segunda enfermedad zoonótica más frecuente, donde $S$. enterica serotipo Enteritidis (SE) fue uno de los serotipos más prevalentes (EFSA 2010). Una situación similar se notificó en Estados Unidos de Norteamérica durante el año 2009 (MMWR 2010). En Chile, en el año 2006 se incrementó en un $300 \%$ el número de casos confirmados de salmonelosis ( 2.219 casos) respecto al año 2000, correspondiendo en el $50 \%$ de los casos al serotipo S. Enteritidis (Figueroa 2007). De la misma forma se observó un aumento en el número de hallazgos de Salmonella spp en mataderos de aves, de 37 en el año 2004 a 74 en el año 2006 (Figueroa 2007).

Aceptado: 01.09.2010.

\# Proyecto Fondecyt 1080291.

* Casilla 2 Correo 15 La Granja, Santiago, Chile; cborie@uchile.cl
Entre los alimentos más frecuentemente asociados se encuentran los derivados de la industria avícola, especialmente el consumo de huevos y sus derivados (Gast 2007). En Chile, entre los años 1975 y 1996, un 38,2\% de los aislados alimentarios de Salmonella Enteritidis se detectaron en alimentos que contenían huevo (tortas, mayonesa), mientras que sólo un $6,4 \%$ de ellos se detectó en alimentos derivados de carne de ave (Prat y col 2001, Figueroa 2007).

Para enfrentar este problema la industria avícola ha implementado varias medidas de control incluyendo vacunas, pre y probióticos, normas de bioseguridad y uso de antimicrobianos, entre otros (Gast 2007). Pese a lo anterior, la enfermedad aún está presente en el ser humano, situación agravada por la emergencia de multirresistencia tanto en las cepas aisladas de casos clínicos como de reservorios animales. Por esta razón, el estudio de bacteriófagos líticos ha concitado el interés en el control de Salmonella gracias a su habilidad para infectar sólo bacterias específicas, manteniéndose inocuos para la microflora intestinal y para células eucariotas. Estos virus o fagos, una vez que reconocen receptores específicos de la superficie bacteriana, inyectan 
su genoma, el cual se multiplica generando una nueva progenie viral; el ciclo termina con la lisis bacteriana y la liberación de la progenie viral al ambiente (Joerger 2003, Dabrowska y col 2005). Gracias a esta actividad lítica, ellos han sido exitosamente usados como herramienta terapeútica y profiláctica frente a patógenos tales como Listeria monocytogenes (Leverentz y col 2003), Campylobacter spp. (Atterbury y col 2003, Bigwood y col 2008), Escherichia coli (O'Flynn y col 2004) y Salmonella spp (Leverentz y col 2001, Modi y col 2001, Whichard y col 2003, Fiorentin y col 2005a, Higgins y col 2005). En el caso específico de Salmonella spp, la fagoterapia ha disminuido la incidencia y el recuento en intestino, así como también la invasión sistémica en pollos (Fiorentin y col $2005^{\mathrm{b}}$, Toro y col 2005, Filho y col 2007). La aplicación profiláctica de bacteriófagos ha producido resultados promisorios. Así, utilizando una mezcla de tres fagos $\left(10^{8}\right.$ Unidades Formadoras de Placas/dosis/fago) en pollos de 6 días de edad pretratados con un probiótico (Broilact@) y luego de tres días, desafiados con SE (2,95 x $10^{5}$ Unidades Formadoras de Colonias/dosis/ave), la incidencia de infección se redujo en $61,3 \%$ y el recuento promedio disminuyó en $0,36 \log _{10} \mathrm{UFC} / \mathrm{g}$ en las aves que recibieron probiótico y fagos, valores que demuestran mayor eficiencia que lo obtenido cuando ambas terapias se administraron por separado (Borie y col 2009).

Los estudios han sido realizados en pollos jóvenes y no existen publicaciones sobre el efecto de una fagoterapia en gallinas de postura, cuyos huevos son considerados alimentos de riesgo para la población humana. Este estudio tuvo como objetivo determinar la eficacia de una mezcla de bacteriófagos en la prevención de la colonización de SE en tejido reproductivo (ovario/oviducto) y ciegos de gallinas comerciales de postura.

\section{MATERIALES Y MÉTODOS}

AVES

Se trabajó con gallinas comerciales de postura Hy-Line Brown de 22 semanas de edad negativas a Salmonella por cultivo y PCR de deposiciones y por prueba serológica de microaglutinación (Servicio Agrícola y Ganadero, Chile). Las aves fueron criadas y manejadas siguiendo las recomendaciones de los Comité de Bioética y Bioseguridad de la Facultad de Ciencias Veterinarias y Pecuarias de la Universidad de Chile y recibieron alimento sin antimicrobianos y agua ad libitum.

\section{CEPA DESAFÍO}

Se utilizó una cepa de SE aislada de "pool" de órganos (hígado, bazo y corazón) de una gallina reproductora. Se seleccionó, en el laboratorio, una mutante espontánea resistente a ácido nalidíxico y rifampicina $\left(n a l^{R} \mathrm{rif}^{R}\right)$.
AISLAMIENTO, PROPAGACIÓN Y CARACTERIZACIÓN DE BACTERIÓFAGOS (BP)

Se seleccionaron tres bacteriófagos líticos previamente caracterizados (Santander 2003, Robeson y col 2008). En breve, $1 \mathrm{~mL}$ de agua de alcantarillado de un plantel avícola de la V Región (zona central de Chile) fue mezclado con $10 \mathrm{~mL}$ de caldo Luria Bertani (LB, Difco) con rifampicina $(100 \mu \mathrm{g} / \mathrm{mL}$, Arlab) y con $0,5 \mathrm{~mL}$ de un cultivo en fase exponencial de crecimiento de SE ATCC 13076 (mutante rifampicina resistente), incubando esta mezcla en agitación a $37^{\circ} \mathrm{C}$ por 24 horas. Un $\mathrm{mL}$ fue centrifugado a $9.300 \mathrm{~g}$ por 5 minutos y el sobrenadante fue tratado con cloroformo y posteriormente sembrado en una placa con desarrollo de SE ATCC 13076 (método de la doble capa de agar). Luego de 24 horas a $37^{\circ} \mathrm{C}$ se purificaron las placas líticas existentes. Se prepararon stocks de bacteriófagos mediante propagación de los fagos purificados en caldo LB con SE ATCC 13076. Este método produjo stocks de fagos con un título $>$ a $10^{12}$ UFP. Cada fago fue administrado vía oral forzada (intraesofágico), suspendido en agua destilada para alcanzar una multiplicidad de infección (MOI) de $10^{3}$ UFP.

\section{DISEÑO EXPERIMENTAL}

Se trabajó con 80 aves de 18 semanas de edad, separadas en cuatro grupos: el grupo 1 constituido por 10 aves que no recibieron terapia ni infección con SE (control negativo), el grupo 2 con 30 aves que recibieron sólo SE (control positivo), el grupo 3 con 10 aves que sólo recibieron bacteriófagos y el grupo 4 con 30 gallinas que fueron tratadas con fagos e infectadas con SE. Los cuatro grupos se mantuvieron en salas experimentales separadas, atendidas por personal exclusivo para evitar contaminación entre ellos. A las 22 semanas de edad, las 10 aves del grupo 3 (sólo BP) y las 30 aves del grupo $4(\mathrm{BP}+\mathrm{SE})$ recibieron por vía oral forzada (intraesofágico) la mezcla de bacteriófagos $\left(10^{11} \mathrm{UFP} /\right.$ dosis/fago $)$ durante tres días consecutivos. Veinticuatro horas más tarde, las 30 aves del grupo 2 (sólo SE) y las 30 aves del grupo 4 fueron desafiadas por vía oral forzada (intraesofágico) con una Dosis Mínima Infectante (DMI) $\left(2,4\right.$ x $10^{8}$ UFC) de SE $n a l^{R}$ rif $^{R}$ suspendida en $3 \mathrm{~mL}$ de agua peptonada fosfatada (Oxoid). Diez días postdesafío, todos los grupos fueron sometidos a eutanasia con T61 (Shering Ploug Animal Health) (AVMA, 2001), obteniendo muestras individuales de ciegos, ovarios y oviductos para análisis de bacteriología cualitativa y cuantitativa. Adicionalmente, en los grupos 2 y 4 se procesaron todos los huevos puestos durante la experiencia (10 días) para la detección de SE, considerando un pool de 10 huevos.

\section{BACTERIOLOGÍA}

Cada muestra de ciego, ovario y oviducto fue pesada y colocada en una bolsa plástica estéril (Whirl Pak, $\mathrm{Nasco}_{\circledast}$ ) 
adicionada de caldo Rapapport-Vassiliadis (RV, Difco) en razón 1:10. Una vez trituradas y homogeneizadas (un $\mathrm{mL}$ fue separado para bacteriología cuantitativa), las muestras se incubaron a $37^{\circ} \mathrm{C}$ por 72 horas, sembrando cada 24 horas en placas de agar XLD (Difco) adicionado de rifampicina y ácido nalidíxico (20 $\mu \mathrm{g} / \mathrm{mL}$ de cada uno) e incubadas a $37^{\circ} \mathrm{C}$ por 24 horas. Las colonias sospechosas se confirmaron con suero anti O-Salmonella poly A-I y Vi (Difco). Las muestras negativas fueron procesadas por PCR para la detección genómica de SE, de acuerdo a metodología implementada y descrita previamente (Borie y col 2008). Los huevos se procesaron como "pool", con un máximo de 10 unidades/"pool"/grupo.

Se consideró un animal positivo cuando se detectó SE al menos en una de sus tres muestras (ciegos, ovario, oviducto).

Para la bacteriología cuantitativa, un $\mathrm{mL}$ de las muestras suspendidas en caldo RV sin incubar fueron diluidas al décimo en agua peptonada fosfatada (Oxoid), sembrando en superficie $100 \mu \mathrm{L}$ sobre agar XLD adicionado de ácido nalidíxico y rifampicina. Las placas se incubaron a $37^{\circ} \mathrm{C}$ por 24 horas, procediendo a contar las unidades formadoras de colonias (UFC).

\section{ANÁLISIS ESTADÍSTICO}

Los resultados de la bacteriología cualitativa fueron expresados como porcentaje de aves infectadas (aves positivas a $\mathrm{SE}$ ) y las diferencias entre los grupos experimentales ( 2 y 4 ) fueron determinadas por Chi cuadrado (INFOSTAT 2004). Los recuentos (UFC) de SE en ciegos y tejidos reproductivos (ovarios/oviductos) fueron transformados a unidades logarítmicas (UFC $\log _{10}$ ) y sometidos a análisis de varianza (ANOVA); las diferencias entre las medias se evaluaron por Test de Tukey (INFOSTAT 2004). Toda muestra que fue positiva en la bacteriología cualitativa pero negativa en la cuantitativa, se le asignó valor 1; por otro lado, toda muestra negativa por bacteriología cualitativa y cuantitativa se le asignó valor 0 . Un valor de $\mathrm{P} \leq \mathrm{a} 0,05$ fue considerado con significancia estadística.

\section{RESULTADOS Y DISCUSIÓN}

El análisis de la bacteriología cualitativa demostró que la proporción de infección cecal y de tejidos reproductivos no fue diferente $(\mathrm{P}>0,05)$ entre el grupo control positivo y el grupo que recibió la mezcla de fagos (cuadro 1). En todas las muestras cecales donde se aisló SE también se detectaron fagos, a diferencia de lo observado en ovarios y oviductos, donde escasamente se aislaron bacteriófagos (datos no mostrados). En ambos grupos experimentales (grupos 2 y 4) no se detectó SE en los huevos frescos puestos durante toda la experiencia (10 días). Todas las muestras de ciegos, ovarios, oviductos y huevos que fueron negativas por bacteriología cualitativa también lo fueron por PCR (datos nos mostrados).
Cuadro 1. Aislamiento de Salmonella Enteritidis desde ciegos, ovarios y oviductos de gallinas de postura experimentalmente infectadas y pretratadas con una mezcla de bacteriófagos.

Isolation of Salmonella Enteritidis from cecum, ovaries and oviducts of experimentally infected laying hens pre-treated with a mixture of bacteriophages.

\begin{tabular}{|c|c|c|c|c|}
\hline \multirow{2}{*}{ Grupo } & \multirow{2}{*}{$\begin{array}{c}\mathrm{N}^{\mathrm{o}} \\
\text { aves }\end{array}$} & \multicolumn{3}{|c|}{ Tejidos positivos a SE (\%) } \\
\hline & & Ciegos & Ovarios & Oviductos \\
\hline Control (SE) & 30 & $29(96,67)$ & $13(43,3)$ & $7(23,3)$ \\
\hline Tratado $(\mathrm{BP}+\mathrm{SE})$ & 30 & $29(96,67)$ & $9(30,0)$ & $3(10,0)$ \\
\hline
\end{tabular}

SE: Salmonella Enteritidis, BP: bacteriófago.

Los promedios de recuentos de SE en ciegos y tejidos reproductivos se observan en la figura 1. En comparación con el grupo control positivo, las aves tratadas con bacteriófagos presentaron una leve disminución de los recuentos, diferencia que fue significativa sólo en ovarios $(\mathrm{P} \leq 0,05)$.

No se observaron cambios de conducta ni manifestaciones clínicas sugerentes de enfermedad digestiva en los grupos experimentales, así como tampoco lesiones macroscópicas en la necropsia.

Bajo el presente diseño experimental la mezcla de bacteriófagos no disminuyó la colonización cecal de SE en las gallinas de postura, situación similar a lo observado por Sklar y Joerger 2001 en pollos tratados con diferentes concentraciones de bacteriófagos y por Higgins y col 2007 en pavos tratados con bacteriófagos asociados a un antiácido. La baja eficiencia de los fagos en gallinas de postura, comparada con los buenos resultados obtenidos previamente en pollos, podría ser explicada por el bajo $\mathrm{pH}$ del tracto digestivo en gallinas adultas comparadas con pollos y mamíferos (Rosales 2005, Ghise 2009). Se ha demostrado que el $\mathrm{pH}$ ácido reduce la actividad lítica de algunos fagos (Leverentz y col 2001, Dabrowska y col 2005), efecto que puede ser revertido por la adición de antiácidos (Smith 1987, Koo y col 2001, Higgins y col 2007). Los fagos utilizados en el presente estudio mantienen un título estable entre pH 3 y 11 (Santander 2003), por lo que no se consideró usar antiácidos. Otros factores adicionales que pudieran incidir en los resultados serían: i) Viscosidad y flora bacteriana normal que actuarían como barrera mecánica, disminuyendo la probabilidad de contacto entre bacterias y fagos (Joerger 2003). ii) motilidad intestinal que disminuiría el tiempo de contacto efectivo entre bacterias y fagos (Fiorentin y col $2005^{\text {b }}$, Atterbury y col 2007, Higgins y col 2007). En este estudio los fagos se administraron 72, 48 y 24 horas antes del desafío con SE, por lo que es factible que los títulos de fagos disminuyeran antes de encontrarse con la bacteria blanco. Para evitarlo, se utilizaron elevadas dosis 


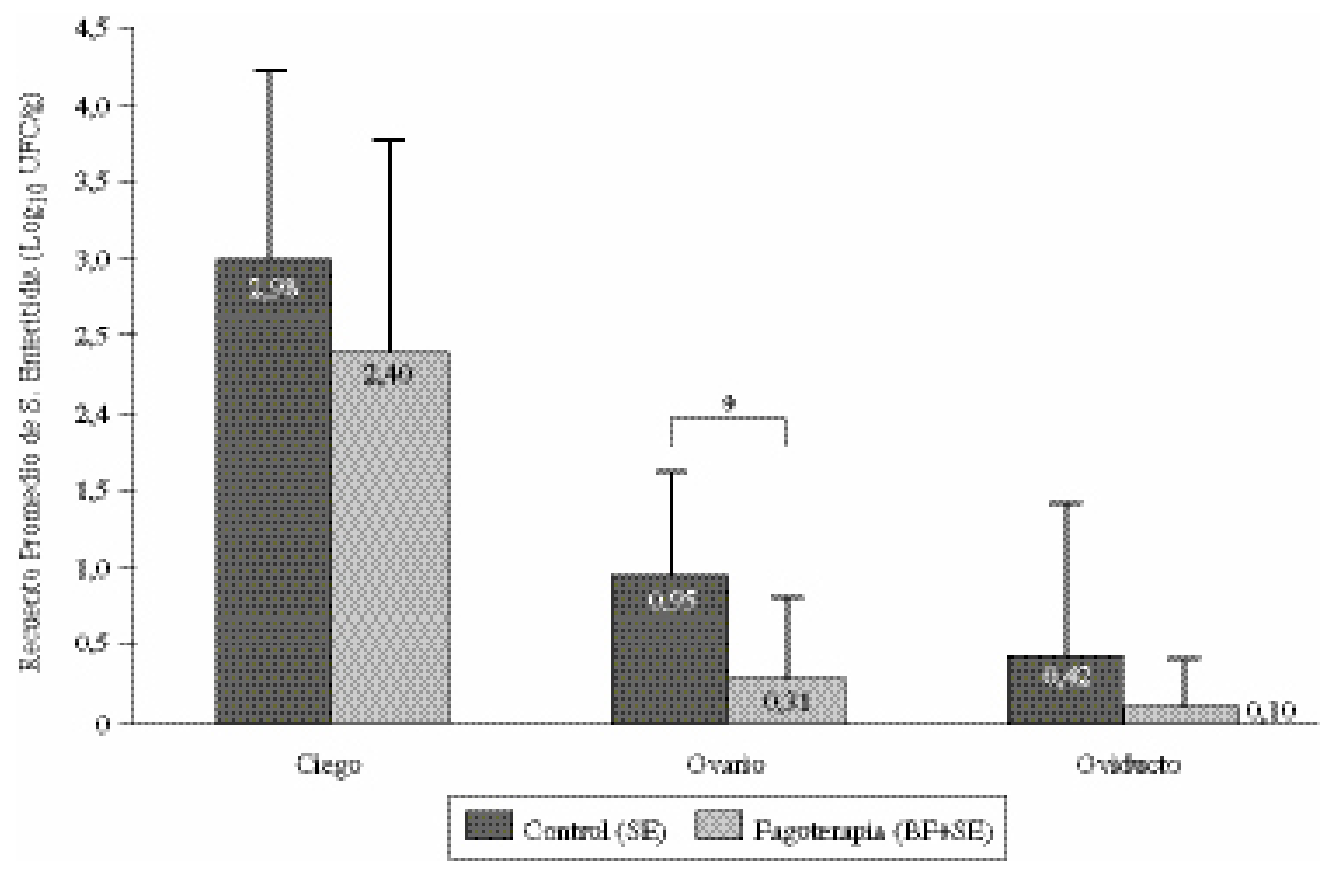

Figura 1. Recuento promedio de Salmonella Enteritidis ( $\mathrm{E}$ ) desde tejidos de gallinas infectadas y pretratadas con bacteriófagos (BF) $(* \mathrm{P} \leq 0,05)$. $(* \mathrm{P} \leq 0.05)$

Average of Salmonella Enteritidis (SE) counts from tissues of chickens experimentally infected and pre-treated with bacteriophages (BF)

de fagos ( $10^{11}$ UFP/día) en relación a la concentración de bacterias inoculadas. iii) Resistencia bacteriana a los fagos (Atterbury y col 2007, Higgins y col 2007), situación que se reduce utilizando dos o más bacteriófagos (Sklar y Joerger 2001, Fiorentin y col 2005a, Toro y col 2005), como es el caso del presente estudio.

La DMI utilizada para infectar las gallinas fue similar a la señalada por otros autores para gallinas adultas (Keller y col 1995, Kinde y col 2000, Gast y col 2004a, Gast y col 2004b). Esta dosis logró una elevada tasa de colonización en ciegos (>95\%) pero escasa en ovarios y oviductos $(43,3 \%$ y $23,3 \%$, respectivamente), esto último probablemente debido a la baja invasividad de la cepa. El escaso aislamiento de SE obtenido en tejidos reproductivos contrasta con lo observado por otros investigadores, donde los porcentajes de aislamiento van de $50-70 \%$ para ovarios y $37-60 \%$ para oviductos (Gast y Beard 1990, Gast y col 2004a). La invasividad de esta cepa podría mejorarse mediante pasajes en tejidos reproductivos de gallinas (Gast y col 2003).

Finalmente, se puede concluir que bajo el presente diseño experimental la administración preventiva de bacteriófagos en gallinas de postura infectadas con SE es menos efectiva que lo descrito previamente en pollos y que, por lo tanto, mayores estudios son necesarios para definir su potencial para disminuir el riesgo en salud pública. El modelo experimental debe ser reevaluado en cuanto a la concentración de bacteriófagos (aumento de la MOI) y a la invasividad de la cepa desafío.

\section{RESUMEN}

Salmonella enterica serotipo Enteritidis (SE) continúa siendo un importante enteropatógeno para la industria avícola y para la Salud Pública. Debido a la limitada efectividad de las medidas de control, los bacteriófagos líticos han mostrado un uso potencial como biocontroladores de SE en aves. El propósito de este estudio fue determinar la efectividad de una terapia profiláctica con fagos para el control de la colonización de SE en el tracto intestinal y reproductivo de gallinas de postura. Gallinas Hy-Line Brown, de 22 semanas de edad, libres de Salmonella, fueron tratadas con una mezcla de tres bacteriófagos ( $10^{11} \mathrm{UFP} /$ dosis/fago $)$ y desafiadas con $2,4 \times 10^{8}$ UFC de $S E$, veinticuatro horas postratamiento. Al día 10 postdesafío, las gallinas se sometieron a eutanasia, obteniéndose muestras individuales de ciegos, ovario y oviducto, las cuales fueron analizadas por bacteriología cualitativa y cuantitativa. Se recolectaron los huevos puestos durante la experiencia y se procesaron para detectar SE. La proporción de Salmonella Enteritidis en los ciegos fue similar entre los grupos control positivo y tratado $(96,67 \%$ ) y el recuento bacteriano cecal tampoco presentó diferencias significativas $(\mathrm{P}>0,05)$. En los tejidos reproductivos, la administración de fagos no disminuyó la incidencia de infección $(\mathrm{P}>0,05)$, pero fue capaz de reducir levemente los recuentos de $\mathrm{SE}$ en ovario $(\mathrm{P} \leq 0,05)$, aunque no en oviducto $(\mathrm{P}>0,05)$. La actividad lítica de los fagos demostrada en tejido ovárico, aunque escasa, sugiere realizar mayores esfuerzos para dilucidar la real contribución de los bacteriófagos como biocontroladores de SE en gallinas de postura.

\section{AGRADECIMIENTOS}

Esta investigación fue financiada por el proyecto Fondecyt $\mathrm{N}^{\mathrm{o}} 1080291$. 


\section{REFERENCIAS}

Atterbury RJ, PL Connerton, C Dodd, CE Rees, IF Connerton. 2003. Application of host-specific bacteriophages to the surface of chicken skin leads to a reduction in recovery of Campylobacter jejuni. Appl Environ Microbiol 69, 6302-6306.

Atterbury RJ, MA Van Bergen, F Ortiz, MA Lovell, JA Harris, A De Boer, JA Wagenaar, VM Allen, PA Barrow. 2007. Bacteriophage therapy to reduce salmonella colonization of broiler chickens. Appl Environ Microbiol 73, 4543-4549.

AVMA, American Veterinary Medical Association. 2001. Report of the AVMA Panel on Euthanasia. J Am Vet Med Assoc 218, 669-696.

Bigwood T, JA Hudson, C Billington, GV Carey-Smith, JA Heinemann. 2008. Phage inactivation of foodborne pathogens on cooked and raw meat. Food Microbiol 25, 400-406.

Borie C, I Albala, P Sánchez, ML Sánchez, S Ramírez, C Navarro, MA Morales, AJ Retamales, J Robeson. 2008. Bacteriophage treatment reduces Salmonella colonization of infected chickens. Avian Dis $52,64-67$

Borie C, ML Sánchez, C Navarro, S Ramírez, MA Morales, J Retamales, J Robeson. 2009. Aerosol spray treatment with bacteriophages and competitive exclusion reduces Salmonella Enteritidis infection in chickens. Avian Dis 53, 250-254.

Dabrowska K, K Switala-Jelen, A Opolski, B Weber-Dabrowska, A Gorski. 2005. Bacteriophage penetration in vertebrates. $J$ App Microbiol 98, 7-13.

EFSA, European Food Safety Authority. 2010. Community Summary Report and Sources of Zoonoses, Zoonotic Agents and food-borne outbreaks in the European Union in 2008. EFSA, Parma, Italy.

Estrada M, S Márquez. 2005. Interacción de los factores ambientales con la respuesta del comportamiento productivo en pollos de engorde. Rev Col Cs Pec 18, 246-257.

Figueroa J. 2007. Descripción y análisis de las acciones realizadas por los servicios públicos (salud animal y salud pública), frente a salmonelosis humana. Memoria de título, Escuela de Ciencias Veterinarias, Universidad de Chile, Santiago, Chile.

Filho R, J Higgins, S Higgins, G Gaona, A Wolfenden, G Téllez, B Hargis. 2007. Ability of bacteriophages isolated from different sources to reduce Salmonella enterica serovar Enteritidis in vitro and in vivo. Poultry Sci 86, 1904-1909.

Fiorentin L, ND Vieira, W Barioni. 2005 . Use of lytic bacteriophages to reduce Salmonella Enteritidis in experimentally contaminated chicken cuts. Braz J Poul Sci 7, 255-260.

Fiorentin L, ND Vieira, WJr Barioni. 2005 . Oral treatment with bacteriophages reduces the concentration of Salmonella Enteritidis PT4 in caecal contents of broilers. Avian Pathol 34, 258-263.

Gast RK, CW Beard. 1990. Isolation of Salmonella enteritidis from internal organs of experimentally infected hens. Avian Dis 34, 991-993.

Gast RK, J Guard-Petter, PS Holt. 2003. Effect of prior serial in vivo passage on the frequency of Salmonella enteritidis contamination in eggs from experimentally infected laying hens. Avian Dis 47, 633-639.

Gast RK, J Guard-Bouldin, PS Holt. 2004a . Colonization of reproductive organs and internal contamination of eggs after experimental infection of laying hens with Salmonella heidelberg and Salmonella enteritidis. Avian Dis 48, 863-869.

Gast RK, BW Mitchell, PS Holt. 2004 . Evaluation of culture media for detecting airborne Salmonella Enteritidis collected with an electrostatic sampling device from the environment of experimentally infected laying hens. Poultry Sci 83, 1106-1111.

Gast RK. 2007. Serotype-specific and serotype-independent strategies for preharvest control of food-borne Salmonella in poultry. Avian Dis 51, 817-828.

Ghise A. 2009. The pH of the Digesta in the Gastrointestinal Tract, in Laying Hens. Bull UASVM, Vet Med 66, 491-492.

Higgins JP, SE Higgins, KL Guenther, W Huff, AM Donoghue, DJ Donoghue, BM Hargis. 2005. Use of a specific bacteriophage treatment to reduce Salmonella in poultry products. Poult Sci 84, 1141-1145.

Higgins S, J Higgins, L Bielke, BM Hargis. 2007. Selection and application of bacteriophages for treating Salmonella enteritidis infections in poultry. Int J Poult Sci 6, 163-168.

Joerger RD. 2003. Alternatives to antibiotics: bacteriocins, antimicrobial peptides and bacteriophages. Poultry Sci 82, 640-647.

Keller L, C Benson, K Krotec, R Eckroade. 1995. Salmonella Enteritidis colonization of the reproductive tract and forming and freshly laid eggs of chickens. Infect Immun 63, 2443-2449.

Kinde H, HL Shivaprasad, BM Daft, DH Read, A Ardans, R Breitmeyer, G Rajashekara, KV Nagaraja, IA Gardner. 2000. Pathologic and bacteriologic findings in 27 -week-old commercial laying hens experimentally infected with Salmonella enteritidis, phage type 4 . Avian Dis 44, 239-248.

Koo J, DL Marshall, A DePaola. 2001. Antacid increases survival of Vibrio vulnificus and Vibrio vulnificus phage in a gastrointestinal model. Appl Environ Microbiol 67, 2895-2902.

Leverentz B, WS Conway, ZAlavidze, WJ Janisiewicz, Y Fuchs, MJ Camp, E Chighladze, A Sulakvelidze. 2001. Examination of bacteriophage as a biocontrol method for Salmonella on fresh-cut fruit: a model study. J Food Protec 64, 1116-1121.

Leverentz B, WS Conway, MJ Camp, WJ Janisiewicz, T Abuladze, MYang, R Saftner, A Sulakvelidze. 2003. Biocontrol of Listeria monocytogenes on fresh-cut produce by treatment with lytic bacteriophages and a bacteriocin. Appl Environ Microbiol 69, 4519-4526.

MMWR, Morbidity and Mortality Weekly Report. 2010. Preliminary FoodNet Data on the Incidence of Infection with Pathogens Transmitted Commonly Through Food-10 states, 2009. $M M W R$ 59, 418-422.

Modi R, Y Hirvi, A Hill, MW Griffiths. 2001. Effect of phage on survival of Salmonella enteritidis during manufacture and storage of cheddar cheese made from raw and pasteurized milk. $J$ Food Protec 64, 927-933.

O’Flynn G, RP Ross, GF Fitzgerald, A Coffey. 2004. Evaluation of a cocktail of three bacteriophages for biocontrol of Escherichia coli O157:H7. Appl Environ Microbiol 70, 3417-3424.

Prat S, A Fernández, A Fica, J Fernández, M Alexandre, I Heitmann. 2001. Tipificación fágica de aislados de Salmonella enteritidis de muestras clínicas, alimentarias y avícolas en Chile. Rev Panam Salud Pública 9, 7-12.

Robeson J, J Retamales, C Borie. 2008. Genomic variants of bacteriophages against Salmonella enterica serovar Enteritidis with potential application in the poultry industry. Braz J Poult Sci 10, 173-178.

Rosales R. 2005. Broilact ${ }^{\circledR}$ hace la diferencia. En: Boehringer Ingelheim Vetmedica S.A. (eds) Una alternativa inteligente: exclusión competitiva. Boehringer Ingelheim Vetmedica S.A, Guadalajara, México.

Santander J. 2003. Aislamiento y caracterización de bacteriófagos biocontroladores de Salmonella enteritidis y su ensayo sobre Salmonella pullorum: evaluación in vivo utilizando el nematodo Caenorhabditis elegans como sistema animal modelo. Tesis de Magíster, Pontificia Universidad Católica de Valparaíso, Valparaíso, Chile.

Sklar I, R Joerger. 2001. Attempts to utilize bacteriophage to combat Salmonella enterica serovar Enteritidis infections in chickens. J Food Safe 2, 15-29.

Smith H, M Huggins, K Shaw. 1987. Factors influencing the survival and multiplication of bacteriophage in calves and in their enviromental. J Gen Microbiol 133, 1127-1135.

Toro H, SB Price, AS McKee, FJ Hoerr, J Krehling, M Perdue, L Bauermeister. 2005. Use of bacteriophages in combination with competitive exclusion to reduce Salmonella from infected chickens. Avian Dis 49, 118-124.

Whichard JM, N Sriranganathan, FW Pierson. 2003. Suppression of Salmonella growth by wild-type and large-plaque variants of bacteriophage Felix $\mathrm{O} 1$ in liquid culture and on chicken frankfurters. J Food Protec 66, 220-225. 